\title{
The Macintosh Laryngoscope: the Mechanism of Laryngeal Exposure and the Optimal Maneuver
}

\author{
Hiroyuki Nakao
}

\begin{abstract}
Background: The Macintosh laryngoscope enables safer and easy intubation. However, the mechanism of laryngeal exposure remains unclear. I hypothesized that extension of the hyoepiglottic ligament contributes to elevation of the epiglottis, and laryngoscope maneuvers that pull the hyoid bone rostrally will effectively elevate the epiglottis and expose the glottides.

Methods: To test this hypothesis, I developed a model in which the epiglottis and hyoid bone were connected with a Velcro tape to allow flexible movement and applied different maneuvers to investigate their effects on epiglottis and hyoid bone movement.
\end{abstract}

Results: A comparison of the original Macintosh maneuver, a modified Macintosh maneuver and the McCoy maneuver found that the McCoy maneuver elevated the epiglottis most, and was associated with rostral and anterior displacement of the hyoid bone. When the model was adjusted so that the hyoid bone was positioned caudal to the epiglottic vallecula, the hyoepiglottic ligament was shortened and the original Macintosh maneuver failed to elevate the epiglottis. Based on these results, a modified Macintosh maneuver was applied that elevated the hyoid bone rostrally and anteriorly and enhanced epiglottis elevation.

Conclusions: The position of the hyoid bone should be considered to achieve epiglottic elevation and a good view of the glottides when performing laryngeal exposure. The position of the hyoid bone relative to the epiglottic vallecula could determine the response of the hyoepiglottic ligament and epiglottis elevation. Increased understanding of the mechanism of laryngeal exposure enables development of improved intubation devices and training models.

Keywords: Hyoepiglottic ligament; Hyoid bone; Epiglottis

\section{Introduction}

R.R. Macintosh developed the Macintosh-type laryngoscope

Manuscript submitted April 3, 2018, accepted May 8, 2018

Department of Emergency and Critical Care, Hyogo College of Medicine, 1-1, Mukogawa, Nishinomiya, Hyogo, 663-8501, Japan.

Email: nakaonakaokobe@yahoo.co.jp

doi: https://doi.org/10.14740/jcs348w in $1943[1,2]$. His revolutionary device and its associated maneuver allow safer and easy intubation. In his original article, Macintosh described a laryngeal exposure maneuver in which the laryngoscope blade tip was inserted into the epiglottic vallecula. It is reported that the epiglottis was elevated by pushing the laryngoscope caudally, in the same direction as the axis of the laryngoscope handle $[1,2]$. However, the mechanism of laryngeal exposure using this approach has not been clarified. Increased understanding of this mechanism should help address difficult intubation cases and assist in development of improved laryngoscope devices.

The mechanism of deglutition is complex, and involves many muscles, ligaments and bones. The function of the epiglottis is to aid in this process, as well as in respiration and olfaction [3]. The hyoepiglottic ligament is a band connecting the hyoid bone to the epiglottis. I hypothesized that extension of the hyoepiglottic ligament would elevate the epiglottis and that laryngoscope maneuvers that pull the hyoid bone rostrally would also contribute to this elevation. The purpose of this study was to elucidate the mechanism by which laryngoscopy maneuvers achieve laryngeal exposure, in terms of the anatomical relationship between the hyoid bone and epiglottis.

\section{Materials and Methods}

\section{The larynx model for intubation}

A model was developed with the anatomical characteristics of the pharynx to investigate the movement of the epiglottis and hyoid bone (Fig. 1a). This model is based on a larynx anatomy teaching model (Kyoto Kagaku Corp, Kyoto city, Japan). A piece of rubber was attached between the hyoid bone and epiglottis using Velcro tape to allow flexible movement of the body of the hyoid bone. The greater horns of the hyoid bone were attached to the thyroid cartilage with short pieces of rubber, also to allow movement of the body of the hyoid bone. The Velcro tape between the epiglottis and hyoid bone represents the hyoepiglottic ligament. The Velcro tape allows adjustment of the length of the hyoepiglottic ligament (Fig. 1b). By changing the length of the Velcro tape, the attachment site of the hyoid bone relative to the epiglottic vallecula can be modified. The model was positioned on a desk in the same orientation as a larynx at intubation.

I assumed that the positional relationship between the epiglottic vallecula and the hyoid bone is important for intuba- 

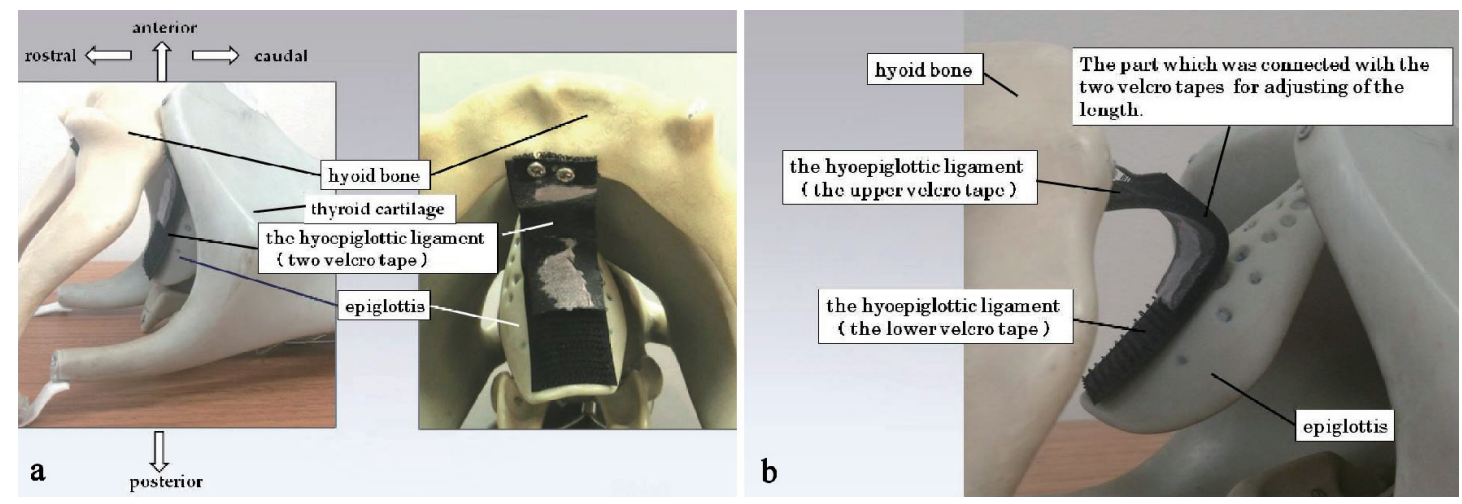

Figure 1. The larynx model for intubation. This larynx model comprises parts representing the thyroid cartilage, hyoid bone, epiglottis and hyoepiglottic ligament. The epiglottis is connected with the hyoid bone via the hyoepiglottic ligament. The epiglottis can be elevated by depression of the hyoepiglottic ligament, according to its anatomical structure (a). In this model, the hyoepiglottic ligament is made of a Velcro tape, the length of which can be adjusted (b).

tion. This study investigated the effects of five different types of maneuvers (models I - V). Number 3 laryngoscope blades were used for all maneuvers. For each maneuver, force was applied to the epiglottis vallecula until no more displacement occurred. To assess the effects of each maneuver in the desktop models, the distance from the desktop to the epiglottis was measured, together with any movement of anatomical structures of the larynx.

The Student's $t$-test $(t$-test, $\mathrm{P}<0.05$; mean $\pm \mathrm{SD})$ was used to compare the distance from the desktop to the epiglottis between each two maneuver.

\section{Study 1}

Two different laryngeal exposure maneuvers (models I and II) were compared. In both, the epiglottic vallecula was positioned caudal to the hyoid bone.

Model I: Application of the original Macintosh laryngoscope maneuver. The laryngoscope blade was inserted into the epiglottic vallecula and the blade elevated caudally, in the direction of the laryngoscope handle, to displace the epiglottis [4].

Model II: Application of the modified Macintosh maneuver. The laryngoscope blade was inserted into the epiglottic vallecula and the hyoid bone elevated anteriorly using the blade tip.

\section{Study 2}

Confirmation that the laryngeal exposure obtained using the McCoy laryngoscope was because of extension of both the hyoepiglottic ligament and hyoid bone (the epiglottic vallecula was positioned caudal to the hyoid bone) [5]. The McCoy laryngoscope is designed so that the blade tip bends upwards when the handle lever is grasped.

Model III: Application of the McCoy maneuver. The blade tip of the McCoy laryngoscope was gently inserted into the epiglottic vallecula and lifted by grasping the lever only, with- out elevating the laryngoscope handle.

\section{Study 3}

It is the confirmation of the role of the hyoid bone in laryngeal exposure.

Model IV: Application of the Macintosh maneuver when the hyoid bone is caudal to the epiglottic vallecula. In many individuals, the hyoid bone is rostral to the epiglottic vallecula (Fig. 2). However, in some cases the hyoid bone is positioned at the same level or caudal to the epiglottic vallecula (Fig. 2a). When the hyoid bone was positioned caudal to the epiglottic vallecula, the hyoepiglottic ligament was very short and the laryngoscope tip could not catch the hyoid bone.

\section{Study 4}

A modified maneuver for maximal laryngeal exposure was used.

Model V: Application of the modified maneuver. A Macintosh laryngoscope blade was inserted into the epiglottic vallecula and the blade tip was lifted anteriorly and rostrally to elevate the hyoid bone.

\section{Study 5}

Unlike Studies 1 - 4, which used the desktop models, this was a preliminary inquiry comparing the Cormack and Lehane classifications of patients after the new maneuver with those after original Macintosh laryngoscope maneuver in medical practice.

In 178 patients, in physical states 1 or 2, general anesthesia was induced by intravenous eslax $(1 \mathrm{mg} / \mathrm{kg})$ and propofol $(2 \mathrm{mg} / \mathrm{kg})$. Initially, we determined the Cormack and Lehane classifications after the original Macintosh laryngoscope maneuver. We then introduced a new maneuver when the Cormack and Lehane classification was determined to be grade 2 or 3 by the original Macintosh laryngoscope maneuver. 


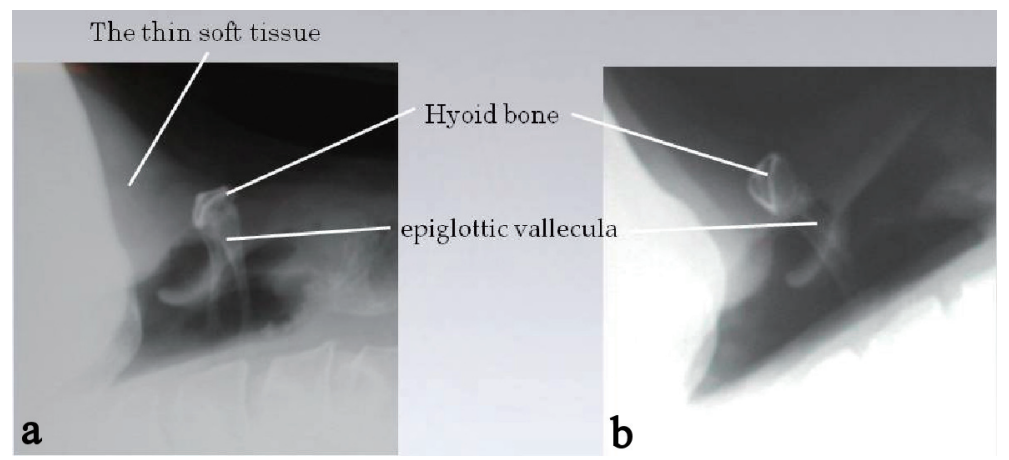

Figure 2. X-ray images of the anatomical positions of the hyoid bone and hyoepiglottic ligament in patients with poor and normal laryngeal exposure. X-ray image from a patient with a poor laryngeal exposure (a). In this patient, the hyoid bone is located caudal to the epiglottic vallecula and the soft tissue under the lower jaw is thick. In many cases the location of the hyoid bone is rostral to the epiglottic vallecula and laryngeal exposure is normal (b).

\section{Results}

\section{Study 1}

Model I: In model I (Fig. 3a), the hyoepiglottic ligament was pushed caudally, which elevated the epiglottis (the distance: $3.0 \pm 0.2 \mathrm{~cm}, \mathrm{~N}=5$ ). However, the supraglottis was covered by the epiglottis. In addition, the thyroid cartilage was pushed caudally in response to strongly pushing the epiglottic vallecula caudally, and the glottides were moved distally, making them harder to observe. The hyoid bone was not displaced.

Model II: The modified Macintosh maneuver used in model II pulled the epiglottis higher (the distance: $3.9 \pm 0.1 \mathrm{~cm}, \mathrm{~N}$ =5) than did the original Macintosh maneuver (Fig. 3b). The hyoepiglottic ligament was also tenser, and the hyoid bone was elevated higher and displaced anteriorly.

\section{Study 2}

Model III: In model III, the bent blade tip of the McCoy laryngoscope pulled the hyoepiglottic ligament rostrally and displaced the epiglottis (the distance: $5.6 \pm 0.1 \mathrm{~cm}, \mathrm{~N}=5$ ), (Fig. $3 \mathrm{c})$. Concurrently, the hyoid bone was elevated rostrally.

\section{Study 3}

Model IV: In this model, it was impossible to displace the hyoid bone or to push the hyoepiglottic ligament using the Macintosh maneuver (maneuver of model I, Fig. 3d). Consequently, it was also impossible to elevate the epiglottis (the distance: $1.5 \pm 0.1 \mathrm{~cm}, \mathrm{~N}=5)$.

\section{Study 4}

Model V: Of the five models, the hyoid bone was raised most anteriorly and rostrally in this model (Fig. 3e). The hyoepiglottic ligament was also most strongly pulled and the epi- glottis was displaced the furthest (the distance: $5.7 \pm 0.1 \mathrm{~cm}$, $\mathrm{N}=5$ ).

There were no significant differences in the distance between model III and V $(\mathrm{P}>0.05)$. There were significant differences in the distance between the other models $(\mathrm{P}<0.05)$.

\section{Study 5}

It was determined that 68 patients were grade 1, 62 were grade 2 and 48 were grade 3 , using the Cormack and Lehane classification and the original Macintosh laryngoscope maneuver. With this maneuver, 62 patients improved from grade 2 to 1 , 19 from grade 3 to 1 and 29 from grade 3 to 2 .

\section{Discussion}

There are no reports about the mechanism underpinning the epiglottis elevation in the laryngeal exposure maneuver described by Macintosh $[1,2]$, and the detailed processes underpinning the development of this maneuver are unknown. The previous direct laryngoscope model blades, the Jackson and the Miller type laryngoscopes, were not inserted into the epiglottic vallecula $[4,6]$. It may be that Macintosh developed his laryngoscope solely based on his clinical experience because the established mechanism of laryngeal exposure is not shown in his article [1].

Surprisingly, the mechanisms underpinning epiglottis elevation have not attracted attention for more than 70 years [7]. In a search of the PubMed database, the search criteria "new laryngoscope" identified 30 articles, while the search criteria "modified laryngoscope" identified 16 articles. However, the rationales for having many types of laryngoscopes, including video laryngoscopes, are not relevant to development of safer intubation devices $[8,9]$. Therefore, it is desirable to clarify the mechanisms of laryngeal exposure to allow the development of new safer intubation devices that are supported by theory.

In study 1, the epiglottis was raised higher in model I than in model II. In model II, the epiglottis was raised $1.0 \mathrm{~cm}$ higher 

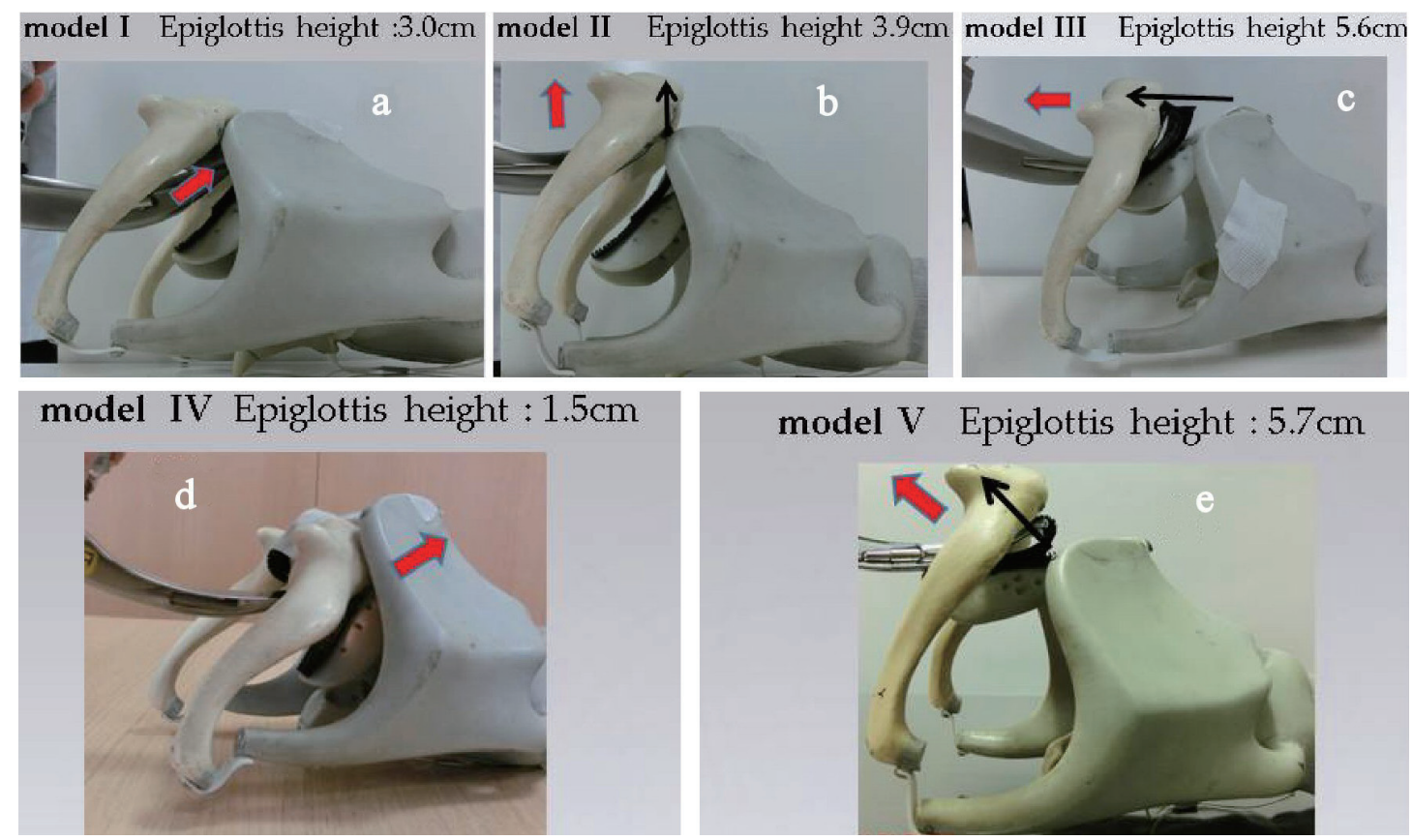

Figure 3. Larynx models showing movement of the hyoid bone and epiglottis. This figure shows a representative image of the maneuver applied to each of the five models. A thick red arrow indicates the direction of force applied through the laryngoscope tip and a small black arrow indicates the direction of movement of the hyoid bone. For each model, the height of the epiglottis above the table surface is described. The epiglottic vallecula is located caudal to the hyoid bone in models I, II, III and V. In model IV the hyoid bone is caudal to the epiglottic vallecula. Prior to each maneuver the epiglottis was $1.5 \mathrm{~cm}$ above the desk. (a) Model I (Macintosh maneuver). The distance between the thyroid cartilage and the hyoid bone was reduced and the thyroid cartilage was at the same level as the hyoid bone. The epiglottis was $2.9 \mathrm{~cm}$ above the desk (partial elevation). The hyoid bone was not displaced. (b) Model II (modified Macintosh maneuver). The distance between the thyroid cartilage and hyoid bone was reduced, but the hyoid bone was displaced anteriorly. The epiglottis was $3.9 \mathrm{~cm}$ above the desk (partial elevation). (c) Model III (the McCoy maneuver). The thyroid cartilage was a little higher than the hyoid bone. The epiglottis was $5.6 \mathrm{~cm}$ above the desk. Displacement of the hyoid bone was in a rostral direction. (d) Model IV (caudal hyoid bone). Prior to the maneuver the location of the hyoid bone was more caudal than in model I. When the maneuver was applied, the epiglottis could not be lifted and remained $1.5 \mathrm{~cm}$ above the desk. This is the same height as when no force was applied. The position of the hyoid bone did not change. (e) Model V (the modified maneuver). The hyoid bone was higher than the thyroid cartilage and displaced anteriorly and rostrally. The epiglottis was $5.8 \mathrm{~cm}$ above the desk.

than in model I, by lifting the hyoid bone anteriorly. In contrast, in model I, the hyoepiglottic ligament was not pulled as much because the hyoid bone was not raised. Thus, it is clear that the modified Macintosh maneuver that displaces the hyoid bone (model II) is more effective than the traction maneuver that pulls the hyoepiglottic ligament alone (the original Macintosh maneuver in model I).

The purpose of model III in study 2 was to confirm the importance of the hyoid bone for tensing the hyoepiglottic ligament. The McCoy laryngoscope blade tip is elevated 70 degrees upwards. McCoy reported that his laryngoscope elevated the hyoepiglottic ligament and exposed the larynx [10].

Clinical studies using the McCoy laryngoscope have reported that it has a higher success rate than the Macintosh laryngoscope in difficult intubation cases [11-16]. Moreover, Wallace reported the McGrath videolaryngoscope can be used as a direct laryngoscopic device in place of the Macintosh. The reason for this is that bent blade tips elevate the hyoid bone and hyoepiglottic ligament [17]. In addition, McCoy reported that use of the McCoy blade requires significantly less force than the Macintosh blade [18]. However, the McCoy laryngoscope is more expensive than the Macintosh; therefore, the Macintosh laryngoscope remains popular. Nevertheless, the McCoy laryngoscope is the best tool to use in difficult airway cases until a videolaryngoscope is available. The results of the present study confirmed the mechanism of the McCoy laryngoscope.

In model IV (study 3), because the blade tip in the epiglottic vallecula was unable to reach the hyoid bone, the epiglottis could not be elevated. In this model, the hyoepiglottic ligament was anatomically very short because the hyoid bone was positioned caudal to the epiglottic vallecula. This situation may be considered as follows. When a string is fixed at one end and free at the other, then, as the string is shortened, the camber is also shorter. In the same manner, it is hard to move the short ligament if force is added to the fulcrum at the blade tip in model V.

The results of studies 1 and 3 investigating anatomical differences in the position of the hyoid bone support the hypothesis that the epiglottis is elevated by tension of the hyoepiglottic ligament by inserting a laryngoscope blade tip. By changing the positional relationship between the hyoid bone and hyoepiglottic ligament using the Velcro tape, a difficult intubation 
scenario can be simulated for training purposes.

The new, more optimized, maneuver involves lifting the hyoid bone and increasing tension on the hyoepiglottic ligament. This maneuver had the highest epiglottis elevation of the five maneuvers investigated. It is not necessary to insert a blade tip into the epiglottic vallecula in this maneuver. Cormack and Lehane classification is a method for grading difficult airways based on the view of the glottis via an inserted laryngoscope. This new optimal maneuver that can lift the epiglottis higher was improved the Cormack and Lehane Grade in study 5. Therefore, it may be beneficial to use this new maneuver with the Macintosh blade in clinical cases where additional epiglottis elevation is desired. This new maneuver is similar to the Backwards Rightwards Upwards Pressure (BRUP) maneuver that is used to improve the laryngoscopic view in difficult intubations [19]. The backward pressure in the BRUP maneuver resembles the anterior traction using the new maneuver in this study, while the upward pressure resembles the rostral traction in the new maneuver. This similarity with the BRUP maneuver further supports the mechanism of the new optimal maneuver and its likely suitability for difficult airways.

No studies of laryngeal exposure have investigated the anatomical variation of the glottis, epiglottis, hyoid bone and hyoepiglottic ligament among individuals. It would be beneficial to investigate the extent of individual anatomical differences and their population frequencies. It was also impossible to determine the force applied using the laryngoscopes to the larynx model in this study. In future, the new optimal maneuver should be clinically tested and compared with the Macintosh maneuver. Additionally, the tensile strength of the model ligament should be compared with that of actual hyoepiglottic ligaments.

It is important to consider the position of the epiglottic vallecula relative to the hyoid bone when elevating the hyoid bone using the Macintosh laryngoscope. For this approach to be effective, it is necessary that the epiglottic vallecula to be located caudal to the hyoid bone so that the blade tip in the epiglottic vallecula lifts the hyoid bone. In these cases, it tends to be difficult to spread the larynx. The Macintosh curved blade applies a force in an anterior and caudal direction. This fact is supported by lifting to hyoepiglottic ligament [20]. However, to elevate the epiglottis most effectively, the new maneuver should be adopted from the present study. Clinical imaging and reports of how the hyoid position correlates with airway difficulty could be used to validate different hyoid positions using this new model. The proportions of individuals with the hyoid bone at different positions would be useful for assessing the clinical significance of the different maneuvers, including the new one.

In conclusion, it is important when performing a laryngeal exposure maneuver to consider the position of the hyoid bone to achieve a better view of the glottides by elevating the epiglottis. Anesthetists should understand the mechanism of laryngeal exposure, and for all clinical cases they should use the maneuver of moving the hyoid bone rostrally. Based on this mechanism, improved intubation devices, training models, and realistic simulations of laryngeal exposure could be achieved. Further validation of this model will determine its suitability for these purposes.

\section{Conflict of Interest}

The author declares that there no conflict of interest regarding the publication of this article.

\section{References}

1. Macintosh RR. A new laryngoscope. The Lancet. 1943;240:205.

2. Jephcott A. The Macintosh laryngoscope. A historical note on its clinical and commercial development. Anaesthesia. 1984;39(5):474-479.

3. Negus VE. The Function of the Epiglottis. J Anat. 1927;62(Pt 1):1-8.

4. Collins SR. Direct and indirect laryngoscopy: equipment and techniques. Respir Care. 2014;59(6):850-862; discussion 862-854.

5. Janeway HH. Intra-tracheal anesthesia from the standpoint of the nose, throat and oral surgeon with a description of a new instrument for catheterizing the trachea. Laryngoscope. 1913;23:1082-1090.

6. Miller RA. A new laryngoscope for intubation of infants. Anesthesiology. 1946;7:205.

7. Burkle CM, Zepeda FA, Bacon DR, Rose SH. A historical perspective on use of the laryngoscope as a tool in anesthesiology. Anesthesiology. 2004;100(4):1003-1006.

8. Bhandari G, Shahi KS, Asad M, Bhakuni R. Airtraq((R)) versus Macintosh laryngoscope: A comparative study in tracheal intubation. Anesth Essays Res. 2013;7(2):232236.

9. Scott J, Baker PA. How did the Macintosh laryngoscope become so popular? Paediatr Anaesth. 2009;19(Suppl 1):24-29.

10. McCoy EP, Mirakhur RK. The levering laryngoscope. Anaesthesia. 1993;48(6):516-519.

11. Ward M. The McCoy levering laryngoscope blade. Anaesthesia. 1994;49(4):357-358.

12. Chadwick IS, McCluskey A. Another trachea intubated with the McCoy laryngoscope. Anaesthesia. 1995;50(6):571.

13. Tuckey JP, Cook TM, Render CA. Forum. An evaluation of the levering laryngoscope. Anaesthesia. 1996;51(1):7173.

14. Laurent SC, de Melo AE, Alexander-Williams JM. The use of the McCoy laryngoscope in patients with simulated cervical spine injuries. Anaesthesia. 1996;51(1):7475.

15. McCoy EP, Mirakhur RK, McCloskey BV. A comparison of the stress response to laryngoscopy. The Macintosh versus the McCoy blade. Anaesthesia. 1995;50(11):943946.

16. Cook TM, Tuckey JP. A comparison between the Macintosh and the McCoy laryngoscope blades. Anaesthesia. 1996;51(10):977-980.

17. Wallace CD, Foulds LT, McLeod GA, Younger RA, McGuire BE. A comparison of the ease of tracheal intubation using a McGrath $\mathrm{MAC}((\mathrm{R}))$ laryngoscope 
and a standard Macintosh laryngoscope. Anaesthesia. 2015;70(11):1281-1285.

18. McCoy EP, Mirakhur RK, Rafferty C, Bunting H, Austin BA. A comparison of the forces exerted during laryngoscopy. The Macintosh versus the McCoy blade. Anaesthe- sia. 1996;51(10):912-915.

19. Knill RL. Difficult laryngoscopy made easy with a "BURP". Can J Anaesth. 1993;40(3):279-282.

20. Macintosh RR. Laryngoscope blades. The Lancet. 1944;243:485. 